\title{
Editorial: Cooperation and Coordination in the Family
}

\author{
James L. Savage ${ }^{1,2 \star}$, Camilla A. Hinde ${ }^{3}$ and Rufus A. Johnstone ${ }^{4}$ \\ ${ }^{1}$ School of Biological, Earth and Environmental Sciences, University College Cork, Cork, Ireland, ${ }^{2}$ Department of Biological \\ Sciences, University of Chester, Chester, United Kingdom, ${ }^{3}$ School of Life Sciences, Anglia Ruskin University, Cambridge, \\ United Kingdom, ${ }^{4}$ Department of Zoology, University of Cambridge, Cambridge, United Kingdom
}

Keywords: cooperation, family conflict, investment, parental care, negotiation

\section{Editorial on the Research Topic}

\section{Cooperation and Coordination in the Family}

\section{INTRODUCTION}

\section{OPEN ACCESS}

Edited and reviewed by: Elise Huchard,

UMR5554 Institut des Sciences de l'Evolution de Montpellier (ISEM), France

*Correspondence: James L. Savage james.savage@cantab.net

Specialty section

This article was submitted to Behavioral and Evolutionary Ecology,

a section of the journal

Frontiers in Ecology and Evolution

Received: 07 October 2020 Accepted: 06 November 2020 Published: 07 December 2020

Citation:

Savage JL, Hinde CA and Johnstone RA (2020) Editorial: Cooperation and Coordination in the Family. Front. Ecol. Evol. 8:614863. doi: 10.3389/fevo.2020.614863
Across diverse taxa, parents face repeated decisions about how to allocate limited resources among their offspring, and must balance investing in their current brood against their future reproductive success. These decisions are still more challenging with multiple carers, as individual contributions are no longer independent: carers must respond to each other in order to partition the work effectively, maximize the benefit of each discrete care event, and avoid being exploited. We refer to this responsiveness as coordination.

Individual care behavior varies - both within and between species-as the social and ecological contexts change (Royle et al., 2012), and we should likewise expect responsiveness to vary. Theoretical work has provided a framework for understanding parental response rules (McNamara et al., 1999; Johnstone and Hinde, 2006; Lessells and McNamara, 2012), but while broad predictions have been borne out, albeit with numerous exceptions (Harrison et al., 2009), the drivers of variation remain unclear. Most existing work has focused on coordination during offspring provisioning in biparental avian systems, but even within this narrow context questions remain around how-and to what extent-responsiveness occurs. Moreover, behavioral response rules are likely to also be important in complex cooperative care groups, in other dimensions of care such as territory defense, and for offspring competing for carer resources. We should expect behavioral rules leading to coordination to minimize costs (e.g., predation risks) and to maximize benefits (e.g., to ensure consistent delivery of care), but have lacked (i) suitable theoretical models of fine-scale responsiveness, (ii) statistical tools for analyzing observed patterns of coordination, and (iii) empirical studies across a range of populations, species and contexts.

The 25 articles in this Research Topic highlight current empirical, theoretical, and comparative research on reproductive coordination, establish what we currently understand and can study effectively, and outline how the questions that remain might be addressed. In this editorial we briefly discuss each contribution in the context of the full collection. 


\section{TURN-TAKING AND SYNCHRONY DURING OFFSPRING PROVISIONING}

Avian provisioning behavior has been extensively studied as a model of parental care. However, carer coordination and responsiveness on the scale of individual provisioning events has only recently attracted significant interest, following work (a) linking provisioning synchrony to reproductive success via reduced predation and/or better food partitioning (e.g., Raihani et al., 2010; Shen et al., 2010; Mariette and Griffith, 2012) and (b) new theory suggesting that turn-taking rules might ameliorate conflict between parents over contributions to care, with better outcomes for parents and offspring (Johnstone et al., 2014). This provisioning coordination proved a popular subject within the Research Topic, which includes a new theoretical model (Johnstone and Savage), a comparative investigation into provisioning synchrony (Khwaja et al.), six articles reporting field studies on coordination between provisioning parents (Burdick and Siefferman; Griffioen et al.; Grissot et al.; Ihle et al.; Lejeune et al.; Story et al.), and three articles primarily concerned with the statistical challenges around inferring responsiveness (Baldan et al.; Ihle et al.; Santema et al.).

The model by Johnstone and Savage extends previous theoretical work on turn-taking by showing that alternation can prove stable even in the face of errors in parents' ability to monitor each other's contributions and asymmetries between parents in the costs and benefits of care. Further, the model suggests that turn-taking, which entails a positive (matching) response to partner effort, can coexist with negative (compensatory) responses over longer time scales.

Taking a comparative approach, Khwaja et al. use nestling provisioning data from 25 Tasmanian and New Zealand passerines to test whether higher nest predation risk favors greater synchrony of parental feeding visits. They find no evidence of greater synchrony in species that evolved with mammalian nest predators, but within one species mainland populations exposed to greater predation in the past c.800 years exhibit higher synchrony than island populations where such predators were never introduced.

Six field studies investigate the relationships between apparent coordination, social/environmental variation, and outcomes for parents and offspring. First, Ihle et al. use data from a longterm study on house sparrows (Passer domesticus) to test whether coordination affects divorce rate, offspring condition, or offspring survival. They find no links between these and either turn-taking or feeding synchrony, and suggest that in this species the observed coordination might be induced by unmeasured environmental variation. Secondly, Lejeune et al. study blue tit (Cyanistes caeruleus) pairs across an altitudinal gradient and varying habitats, again measuring both synchrony and alternation. They find that lower-altitude pairs alternate more than those at higher altitudes, and synchrony increases for pairs occupying woodland-pasture edges compared to interiors. Furthermore, while there is no effect of coordination on fledging success, in woodland habitats more synchronous pairs have heavier chicks. Griffioen et al. also studied blue tits, but experimentally manipulated provisioning behavior through clipping the feathers of male parents. They surprisingly find no change in visit rate or turn-taking behavior in handicapped males, but do detect an increase in visit rate and a reduction in turn-taking in the unmanipulated partner, illustrating parents' ability to adjust their contributions to their partner's state. Fourthly, in an observational study Burdick and Siefferman investigate alternation in eastern bluebird (Sialia sialis) pairs across spatial variation in interspecific competition. Increasing density of competing species reduces provisioning rate and reproductive success but does not affect alternation. However, under high competitor density, nestlings grow faster if their parents alternate more.

In contrast to the relatively fast-provisioning passerine examples above, Grissot et al. present a study of parental coordination in the little auk (Alle alle), a planktivorous arctic seabird with a dual foraging strategy of short and longer trips to feed chicks and themselves, respectively. Across five breeding seasons, parents coordinate by avoiding simultaneous long trips, facilitating a less variable distribution of food delivery to chick. Coordination varies substantially between pairs in every season, despite shared environmental conditions, and is more pronounced under beneficial environmental conditions that facilitate the delivery of higher-energy food loads. However, greater coordination does not appear to increase chick body condition. Finally, Storey et al. explore the behavioral rules underpinning provisioning behavior in Common Murres (Uria aalge), in which parents typically return to the nest with food and then take over brooding the chick. Variation in resource availability mediates the likelihood that parents attempted to relieve each other without feeding, with high-provisioning parents more sensitive to this variation. The authors conclude that both parents negotiate parental duties as conditions change, but that high-quality partners are in better condition and hence more able to compensate for their partners when resources are abundant.

Interpreting the observed patterns of turn-taking and synchrony in carer visits can be challenging, in part because natural refractory periods (Johnstone et al., 2014), different numbers of carers (Savage et al., 2017), and environmental variability (Schlicht et al., 2016) can result in suggestive patterns without direct responsiveness occurring. Three Research Topic articles explore this topic using simulations: first, Santema et al. illustrate the limitations of using randomized visit intervals to calculate the expected degree of alternation when parents are not coordinating, through simulating data in which parental visit rates simultaneously change during part of the observed period (correlated temporal heterogeneity). This effect, which could be driven by shared environmental factors such as a nearby predator, is further explored by Ihle et al., who also illustrate how certain metrics of alternation and synchrony are linked to the relative visit rates of the carers. Ihle et al. then evaluate five candidate models for analyzing alternation, and use their preferred model to explore an example dataset on 
house sparrows (Passer domesticus), finding no brood or pair characteristics that predicted variation in alternation. Finally, Baldan et al. use simulations and empirical data from great tit (Parus major) pairs to identify the degree of observed alternation attributable to correlated temporal heterogeneity. Their novel approach uses "pseudo-pairs" during randomizations to calculate expected levels of alternation, in which individual visit data is matched with contemporaneous data from oppositesex individuals at other, nearby nests. Baldan et al. infer that around $18 \%$ of observed alternation is attributable to temporal heterogeneity affecting different pairs simultaneously. All three studies call for increased care when interpreting patterns of visit data, and for further experimental studies to more conclusively exclude alternative explanations for synchrony and turn-taking. We wholeheartedly agree with both of these positions.

\section{DIVERSITY OF CARE AND COORDINATION BEHAVIOR WITHIN THE FAMILY}

Provisioning young is not the only context in which coordination occurs, and seven articles within this Research Topic address other modes of care and familial interactions. First, Savage and Hinde review how care and coordination are quantified, and Pogány et al. investigate the origin of coordination rules by studying the long-term effects on incubation and provisioning behavior of nestlings raised by single parents. The remaining articles focus on different stages of care such as incubation (Bulla et al.) or post-fledging (Franks et al.), and on the importance of vocal behavior in mediating coordination (Ducouret et al.; Mariette).

The mini-review by Savage and Hinde attempts to categorize the range of analyses around parental care into three distinct types: (a) the temporal distribution of care behavior, such as variance, repeatability, and differences across stages, (b) variation among care events, such as load size, false-feeding, and favoritism, and (c) interactions between carers, such as turntaking, synchrony, and task specialization. They advocate for broader use of these analyses rather than a focus on simple rates of care, and note the need for a diversity of studies, particularly on behavior beyond provisioning.

The complex origins of care and coordination behavior are illustrated by Pogány et al., who use a split-family design to produce fledgling zebra finches (Taeniopygia guttata) raised by either their mother or father alone, and find strong short-term effects of increased begging and preference toward their caring parent. Furthermore, when these fledglings mature, their behavior differs during the incubation period of their first breeding attempt, but not during provisioning or during their second breeding attempt, suggesting that effects of early social experiences can be overwritten by later experiences.

In a direct investigation into response rules during incubation, Bulla et al. present a 12-h partner removal experiment on sandpipers (Calidris pusilla), monitoring nest attendance of the remaining partner. The responses of remaining partners of both sexes range from no compensation to full compensation, potentially because of variation in individual reserves and environmental conditions. Partners do not appear to retaliate after the removed individual returns, and although nest attendance reduces after the experiment this gradually returns to normal.

Focusing instead on the post-fledging period, Franks et al. use Passive Integrated Transponder tags and feeding stations to investigate coordination in hihi (Notiomystis cincta), an endangered New Zealand passerine. Fledglings in poorer condition follow parents to feeders more closely, and broods in poorer condition disperse earlier from the natal territory. However, neither overwinter survival nor number of associates as a juvenile are predicted by this variation as a fledgling.

The final two articles address familial interactions mediated through vocalizations. In a perspective article, Mariette first discusses vocal communication between parents jointly rearing offspring, including its function in signaling individual state, then addresses parent-offspring communication including recent work on acoustic communication between parents and embryos and its potential for developmental programming. Mariette concludes with vocal communication between siblings in the contexts of synchronized hatching and negotiations over food distribution, suggesting that vocal interactions are a key mechanism for negotiation and coordination that will require novel empirical acoustic research to understand. Providing an example of such research, Ducouret et al. present a study of vocal negotiation in nestling barn owls (Tyto alba), using a novel "automatic interactive playback" methodology to interact with individual nestlings in real time. They show that matching an opponent's call duration and mismatching their call rate are the most effective strategies for dominating the interaction, and that these strategies were also costlier, suggesting that signals are honest. These results underline the importance of real-time interactions, and highlight the potential of interactive playbacks to understand coordination.

\section{COOPERATION AND COORDINATION OVER LONGER TIMESCALES}

Studies of familial cooperation and coordination have often focused narrowly on the provision of care in a single family or breeding group during a single reproductive attempt. However, it is increasingly clear that cooperative behavior may vary substantially across years or breeding attempts, and within and between groups in any 1 year. Breeding episodes are typically embedded within a longer-term history of cooperative interaction, and awareness of this wider context may inform the costs and benefits of care (or related behaviors) and the nature of the relationship between family members. A number of the contributions to this Research Topic explore these broader and longer-term contexts, through studies of individual species (Koenig et al.; Nomano et al.; Pike et al.), comparative analyses across species (Wagner et al.), and the 
implications for our general understanding of the evolution of family interaction (Griffith).

The extent to which cooperative behavior may vary across individuals and breeding attempts is demonstrated by Pike et al., who study investment in offspring care by helpers and breeders in the Western Australian magpie (Cracticus tibicen dorsalis). Across multiple groups over two consecutive breeding seasons, they report significant variation in care effort, which is strongly influenced by group size, chick age, and by helper traits such as age, sex, and foraging efficiency. Nomano et al. similarly examine contributions to nestling care in the chestnut-crowned babbler (Pomatostomus ruficeps), and show that in this species the pattern of provisioning is highly sensitive to meteorological conditions; on days with high wind speed, for instance, dominant males contributed less, as did helpers, who also showed reduced visit rates on days with high mean temperature. Moreover, large breeding groups visited the nest more asynchronously on warmer days, showing that weather conditions affect not only individual caring effort, but also the degree of grouplevel coordination. Lastly, Koenig et al. document variation in cooperative effort in the acorn woodpecker (Melanerpes formicivorus), and further explore the fitness benefits conferred by male and female helpers, and how these vary across multiple groups and years. They show that the effects of male helpers increase with food availability, specifically the size of the prior year's acorn crop. Intriguingly, while the time that helpers spend tending acorn-storing granaries shows a similar pattern, the rate of feeding at the nest does not. The authors argue that the benefits conferred by helpers may therefore reflect behaviors other than feeding. Together, these studies highlight how studying helping across multiple reproductive attempts, and under varying environmental conditions, can yield novel insights into the evolutionary significance of helping and cooperative care.

Turning to cross-species analysis, Wagner et al. report the results of handicapping experiments conducted on five different altricial bird species, using the same experimental treatment to facilitate comparison. The authors report that across all species, handicapped individuals reduce their nest visitation rates, but that their partners generally do not compensate for this reduction, so that costs are passed on to the offspring. This effect, however, is more marked in species with long care periods, though independent of life history pace. The nature of the interaction among carers, and the extent and details of their coordination, thus vary across species in a way that is sensitive to the costs and benefits of caring.

Lastly, Griffith contributes a general discussion of cooperation and coordination in monogamous birds, in which he argues that, given the long-term nature of socially monogamous bonds in many avian species, biologists have over-emphasized the importance of evolutionary conflict between mates. Since males and females clearly gain from establishing and maintaining socially monogamous partnerships, often persisting through many reproductive attempts, greater attention should be focused on the fitness benefits of a successful partnership, the traits and processes that contribute to the formation of such relationships, and the ecological and evolutionary conditions that favor them.

\section{BROADER TAXONOMIC PERSPECTIVES}

Research into familial coordination has been dominated by studies of birds. This emphasis is no less apparent in this Research Topic, in which the great majority of empirical papers focus on avian study systems. However, cooperation and coordination can be observed and studied in other taxa, as illustrated by contributions on fishes (Taborsky and Riebli), insects (Smiseth), and arachnids (Junghanns et al.). The latter two cases follow many avian studies by focusing on investment in offspring provisioning, but in different modes: regurgitation feeding and sacrifice of a female's body as food for offspring in suicidal care. The former case illustrates the contrasting behaviors of cooperation through joint shelter excavation and territory defense.

While it is often assumed that complex strategies of negotiation and coordination, such as turn-taking and reciprocity, are limited in their taxonomic distribution, Taborsky and Riebli demonstrate that daffodil cichlids (Neolamprologus pulcher) are capable of both temporal coordination and contingent, sequential cooperation. Indeed, they argue that their study provides the first experimental evidence for the latter behavior in a cooperatively breeding vertebrate. Turning to cooperative care in burying beetles, Smiseth observes that despite little explicit study of the distribution of parental provisioning over time, there is nevertheless clear evidence for negotiation over care in this species, with parents adjusting their own investment in response to that of their partner. Moreover, recent studies have highlighted the complexity of this process, showing that parents can independently adjust their own caring effort based on both their partner's contribution to care and on direct assessment of its state, and that negotiation may extend to coordination of food consumption as well as provisioning of young. Lastly, Junghanns et al. (2019) demonstrate that (like mothers) non-reproductive helpers in the cooperatively breeding spider Stegodyphus dumicola undergo irreversible physiological adaptation to facilitate offspring provisioning, highlighting the need to consider the physiological "preparation" for care in discussions of familial cooperation, as well as overt care behavior itself.

Collectively, these three contributions show the value of a wider taxonomic focus in studies of familial coordination. As well as a broader range of examples across different forms of care and cooperation, such studies can also draw attention to aspects of negotiation that have received little or no attention in avian studies.

\section{CONCLUSIONS}

Recent interest in behavioral coordination and responsiveness during parental care, culminating in this Research Topic, has generated a clear theoretical and empirical basis for further research. While observations of parental responsiveness are now widely reported (at least in birds), there remain questions around the interpretation of the patterns of care observed, and more experimental studies manipulating short-term care are 
desirable. There remains a focus on avian provisioning behavior, presumably because of the prevalence of biparental care in birds and the relative ease of data collection, despite other forms of coordination being both common and carrying substantial fitness consequences. We particularly encourage further work on nonavian species, and on behaviors other than offspring provisioning at a fixed location, to facilitate a more general understanding of coordination behavior.

\section{AUTHOR CONTRIBUTIONS}

JS and RJ wrote the manuscript. JS, $\mathrm{CH}$, and RJ edited the manuscript and administered the Research Topic collection.

\section{REFERENCES}

Harrison, F., Barta, Z., Cuthill, I. C., and Székely, T. (2009). How is sexual conflict over parental care resolved? A meta-analysis. J. Evol. Biol. 22, 1800-1812. doi: 10.1111/j.1420-9101.2009.01792.x

Johnstone, R. A., and Hinde, C. A. (2006). Negotiation over offspring carehow should parents respond to each other's efforts? Behav. Ecol. 17, 818-827. doi: 10.1093/beheco/arl009

Johnstone, R. A., Manica, A., Fayet, A. L., Stoddard, M. C., Rodriguez-Gironés, M. A., and Hinde, C. A. (2014). Reciprocity and conditional cooperation between great tit parents. Behav. Ecol. 25, 216-222. doi: 10.1093/beheco/ art109

Lessells, C. M., and McNamara, J. M. (2012). Sexual conflict over parental investment in repeated bouts: negotiation reduces overall care. Proc. R. Soc. B 279, 1506-1514. doi: 10.1098/rspb.2011.1690

Mariette, M. M., and Griffith, S. C. (2012). Nest visit synchrony is high and correlates with reproductive success in the wild zebra finch taeniopygia guttata. J. Avian Biol. 43, 131-140. doi: 10.1111/j.1600-048X.2012.05555.x

McNamara, J. M., Gasson, C. E., and Houston, A. I. (1999). Incorporating rules for responding into evolutionary games. Nature 401, 368-371. doi: 10.1038/4 3869

Raihani, N. J., Nelson-Flower, M. J., Moyes, K., Browning, L. E., and Ridley, A. R. (2010). Synchronous provisioning increases brood survival in cooperatively breeding pied babblers. J. Anim. Ecol. 79, 44-52. doi: $10.1111 /$ j.1365-2656.2009.01606.x
All authors contributed to the article and approved the submitted version.

\section{FUNDING}

JS was supported by an Irish Research Council Postdoctoral Fellowship.

\section{ACKNOWLEDGMENTS}

We would like the thank all authors and reviewers that contributed to this Research Topic, and the staff at Frontiers in Ecology and Evolution for invaluable administrative support throughout the process.
Royle, N. J., Smiseth, P. T., and Kölliker, M. (2012). The Evolution of Parental Care. Oxford: Oxford University Press. doi: 10.1093/acprof:oso/9780199692576.001.0001

Savage, J. L., Browning, L. E., Manica, A., Russell, A. F., and Johnstone, R. A. (2017). Turn-taking in cooperative offspring provisioning: by-product of individual provisioning behaviour or active response rule? Behav. Ecol. Sociobiol. 71:162. doi: 10.1007/s00265-017-2391-4

Schlicht, E., Santema, P., Schlicht, R., and Kempenaers, B. (2016). Evidence for conditional cooperation in biparental care systems? A comment on Johnstone et al. Behav. Ecol. 27, e2-e5. doi: 10.1093/beheco/arw036

Shen, S.-F., Chen, H.-C., Vehrencamp, S. L., and Yuan, H.-W. (2010). Group provisioning limits sharing conflict among nestlings in joint-nesting Taiwan yuhinas. Biol. Lett. 6, 318-321. doi: 10.1098/rsbl.2009.0909

Conflict of Interest: The authors declare that the research was conducted in the absence of any commercial or financial relationships that could be construed as a potential conflict of interest.

Copyright (c) 2020 Savage, Hinde and Johnstone. This is an open-access article distributed under the terms of the Creative Commons Attribution License (CC BY). The use, distribution or reproduction in other forums is permitted, provided the original author(s) and the copyright owner(s) are credited and that the original publication in this journal is cited, in accordance with accepted academic practice. No use, distribution or reproduction is permitted which does not comply with these terms. 